\title{
Low-dose Bupivacaine with Dexmedetomidine Prevents Hypotension After Spinal Anesthesia
}

\author{
Young Seop Chang ${ }^{1}$, Ji-Eun $\mathrm{Kim}^{2}$ and Tae-Yun Sung, ${ }^{2, *}$ \\ ${ }^{I}$ Department of Urology, Konyang University Hospital, Konyang University College of Medicine, Daejeon, Korea; \\ ${ }^{2}$ Department of Anaesthesiology and Pain Medicine, Konyang University Hospital, Konyang University College of \\ Medicine, Daejeon, Korea
}

\begin{abstract}
Background: We assessed whether intrathecal low-dose bupivacaine with dexmedetomidine could reduce the incidence of spinal anesthesia (SA)-related hypotension. Methods: In total, 47 patients undergoing urological or orthopedic surgery under SA were randomized into two groups. SA was induced using $12.5 \mathrm{mg}$ of hyperbaric bupivacaine alone (Group B; $n=24$ patients) or $6 \mathrm{mg}$ of hyperbaric bupivacaine plus $4 \mu \mathrm{g}$ of dexmedetomidine and $0.3 \mathrm{ml}$ of saline (Group $\mathrm{BD} ; n=23$ patients). At $10 \mathrm{~min}$ after SA, dexmedetomidine was infused in both groups at a loading dose of $0.5 \mu \mathrm{g} / \mathrm{kg}$, administered over $10 \mathrm{~min}$, and then maintained at a dose of $0.2 \mu \mathrm{g} / \mathrm{kg} / \mathrm{h}$ for $40 \mathrm{~min}$. Results: The incidence of hypotension was significantly higher in Group B than in Group BD $(50.0 v s .17 .4 \%, P=0.018)$. The amount of ephedrine used to treat hypotension was significantly higher in Group B than in Group BD (median [range], 3 [0-30] vs. 0 [0-12] mg, $P=0.014$ ). Conclusion: Low-dose bupivacaine plus dexmedetomidine SA reduced the incidence of hypotension compared to conventional bupivacaine SA.
\end{abstract}

Keywords: Bupivacaine, dexmedetomidine, hypotension, spinal anesthesia.

\section{INTRODUCTION}

Despite the advantages of spinal anesthesia (SA) compared with general anesthesia, including a lower cost, better postoperative respiratory function, and the elimination of complications due to intubation and extubation, hypotension and intraoperative discomfort due to patient anxiety and/or inadequate sedation after SA remain common problems [1]. Thus, to reduce the incidence of hypotension after SA, lowdose intrathecal bupivacaine with opioids has been studied and showed a significantly reduced incidence of hypotension $[2,3]$. However, low-dose local anesthetics can increase the risk of an inadequate block level [2], and intrathecal opioids can cause several problems, including respiratory depression, pruritus, and central nervous system excitation [4].

Dexmedetomidine is an $\alpha_{2}$-adrenoreceptor agonist that has been used mainly as an intravenous sedation agent and co-analgesic drug in regional anesthesia or for patients who require invasive procedures or mechanical ventilation care in an intensive care unit. Apart from its sedative and analgesic effects, it has anxiolytic, antishivering, and respiratory stabilizing properties [5]. In previous studies, intravenous dexmedetomidine with spinal anesthetics provided sufficient sedation and anxiolysis and prolonged the duration of SA without causing significant hemodynamic instability [6]. Also, intrathecal dexmedetomidine, as an adjuvant to intrathecal local anesthetics, provided analgesic properties and prolonged the duration of the motor and sensory block without neurological impairment [7].

\footnotetext{
*Address correspondence to this author at the 158 , Gwangeodong-ro, Seogu, Department of Anesthesiology and Pain Medicine, Konyang University Hospital, Konyang University College of Medicine, Daejeon, 302-718, Korea; Tel: +82-42-600-9316; Fax: +82-42-545-2132;

E-mail: unt1231@naver.com
}

Although it is known that both intravenous and intrathecal dexmedetomidine administration can prolong the sensory and motor block in SA, no study has reported the impact of low-dose spinal bupivacaine with concurrent administration of low-dose intrathecal and intravenous dexmedetomidine on hemodynamic and sedation effects.

We hypothesized that intrathecal low-dose bupivacaine with dexmedetomidine plus an intravenous dexmedetomidine infusion would provide an adequate quality of anesthesia and reduce the incidence of hypotension. Thus, we compared the hemodynamic and anesthetic effects of intravenous dexmedetomidine infusion after a spinal injection of low-dose $(6 \mathrm{mg})$ bupivacaine with dexmedetomidine $(4 \mu \mathrm{g})$ versus a conventional spinal dose $(12.5 \mathrm{mg})$ of bupivacaine in patients undergoing urological or orthopedic surgery under SA.

\section{METHODS}

\section{Study Population}

This prospective, randomized, controlled trial was approved by the Konyang University Hospital Institutional Review Board, Daejeon, Republic of Korea, and registered at http://cris.nih.go.kr/ (KCT 0001064). Written informed consent was obtained from American Society of Anesthesiologists physical status I-II patients undergoing elective urologic or orthopedic surgery under SA before the study began.

Patients who had contraindications to SA such as coagulopathy or other bleeding diathesis, infection at the injection site for SA, or a history of hypersensitivity to local anesthetics, and those who were younger than 20 years old or had a history of arrhythmia or uncontrolled hypertension were excluded from this study. 
Before anesthesia induction, each patient was given a sealed envelope indicating his group assignment. The patients were randomly allocated (allocation ratio $=1: 1$ ) to undergo the procedure either in the bupivacaine group (Group B) or in the low-dose bupivacaine and dexmedetomidine group (Group BD).

\section{Anesthesia and Measurements}

All patients arrived at the operating suite with no premedication. Before SA, pulse oximetry, electrocardiography, and noninvasive blood pressure monitoring were started. Noninvasive automated blood pressure measurements were recorded at 2.5-min intervals until the end of the loading dose infusion of dexmedetomidine, then every $5 \mathrm{~min}$ during surgery, and then every $15 \mathrm{~min}$ in the post-anesthesia care unit (PACU) until discharge to the general ward. Oxygen (6 $1 /$ min) was supplied via a facemask and end-tidal carbon dioxide was monitored. Before SA, $500 \mathrm{ml}$ of crystalloid fluid was supplied via a peripheral vein with an 18-gauge angio-catheter. Dexmedetomidine (Precedex, $200 \mu \mathrm{g} / 2 \mathrm{ml}$; Hospira, Inc., Lake Forest, IL) was mixed with $48 \mathrm{ml}$ of preservative-free normal saline $(4 \mu \mathrm{g} / \mathrm{ml})$ and prepared for both intrathecal injection and continuous intravenous infusion. Baseline mean blood pressure was determined from the average of three consecutive readings taken after administration of the crystalloid fluid.

SA was conducted in the lateral decubitus position with the involved side down. The subarachnoid space was punctured with a 25-gauge Quincke needle via the L4/5 intervertebral space using a midline approach. After confirming the intrathecal position of the needle by the leakage of cerebrospinal fluid, the intrathecal anesthetics were introduced: 2.5 $\mathrm{ml}$ of hyperbaric bupivacaine $0.5 \%(12.5 \mathrm{mg})$ for Group B and $1.2 \mathrm{ml}$ of hyperbaric bupivacaine $0.5 \%(6 \mathrm{mg})$ with $1 \mathrm{ml}$ of dexmedetomidine $(4 \mu \mathrm{g})+0.3 \mathrm{ml}$ of preservative-free normal saline for Group BD. The injected volume in both groups was $2.5 \mathrm{ml}$. The intrathecal anesthetics were slowly injected over 20 seconds in both groups. The intrathecal anesthetics were prepared by a registered nurse blinded to the study. SA in all patients was conducted and managed by an anesthesiologist who was blinded to both the study and the intrathecal anesthetic procedure. After administration of the intrathecal anesthetics, the patient's position was changed to a supine position.

The sensory and motor block level was assessed $10 \mathrm{~min}$ later in the supine position using a pinprick test and the modified Bromage scale (MBS; $0=$ no paralysis, $1=$ unable to raise extended leg, 2 = unable to flex knee, and $3=$ unable to flex ankle) [8], respectively. At $10 \mathrm{~min}$ after the spinal injection, the inability to reach a sensory block at T12 and an MBS score of 0 was considered a block failure and the subject was excluded from further study.

Following the sensory and motor block level assessment, intravenous dexmedetomidine was infused in both groups at a loading dose of $0.5 \mu \mathrm{g} / \mathrm{kg}$ over $10 \mathrm{~min}$, and then continuously infused at a dose of $0.2 \mu \mathrm{g} / \mathrm{kg} / \mathrm{h}$ for $40 \mathrm{~min}$. Considering the elimination half-life of dexmedetomidine $(\sim 2 \mathrm{~h})$ [5] and the planned duration of the study procedures $(\sim 50-80$ $\mathrm{min})$, the infusion duration was planned to be $50 \mathrm{~min}$. The level of sedation was assessed immediately before the dexmedetomidine loading dose infusion (baseline) and every 10 min during dexmedetomidine continuous infusion, and then every 15 min using the Ramsay sedation scale $(1=$ patient anxious, agitated, or restless; 2 = patient cooperative, oriented, and tranquil alert; 3 = patient responds to commands; $4=$ asleep, but with brisk response to light glabellar tap or loud auditory stimulus; 5 = asleep, with sluggish response to light glabellar tap or loud auditory stimulus; and $6=$ asleep, with no response) [9] until the patient was discharged from the PACU.

Hypotension was defined as a decrease in systolic blood pressure (SBP) of $>25 \%$ of the preanesthetic SBP or an SBP $<100 \mathrm{mmHg}$ during the $3 \mathrm{~h}$ after the induction of SA. Hypotension was treated with crystalloid fluid replacement (intravenous, 50-100 ml) and a bolus of ephedrine (6 mg). Bradycardia was defined as a heart rate $<50 \mathrm{bpm}$ and was treated with intravenous atropine $(0.5 \mathrm{mg})$.

All operations were performed by surgeons blinded to the study. All patients were observed for at least $3 \mathrm{~h}$ after SA. Adverse effects were recorded by a registered nurse who was blinded to the study.

\section{Statistical Analysis}

Data were analyzed using SPSS software (ver. 18.0 for Windows; SPSS Inc., Chicago, IL, USA). On the basis of a previous study [3], a sample size of 22 patients per group was determined through a power analysis $(\alpha=0.05, \beta=$ 0.90 ) to detect a mean difference in SBP of $20 \mathrm{mmHg}$ with a standard deviation of $20 \mathrm{mmHg}$ at $10 \mathrm{~min}$ after SA. Thus, considering a potential dropout rate of $10 \%$, we enrolled 24 patients per group.

The normal distribution of continuous variables was analyzed using the Kolmogorov-Smirnov test. Continuous variables were compared using an unpaired two-tailed $t$-test or the Mann-Whitney $U$ test and are reported as means \pm standard deviation or medians (range), as applicable. Categorical variables were compared using the $\chi^{2}$ test or Fisher's exact test, as applicable.

SBP over time was compared between the groups using variance analysis for repeated measures, followed by Student's $t$-test. Ramsay sedation scales over time and changes from baseline between the groups were compared using variance analysis for repeated measures, and a post hoc analysis with the Bonferroni method was used for multiple pair-wise comparisons. $P$-values $<0.05$ were considered statistically significant.

\section{RESULTS}

During the study, there were 55 cases of SA. Of them, seven were excluded: two because of age ( $<20$ years old), three because of a history of arrhythmias, and two because they declined to participate. In total, 48 patients began the study but one patient in Group BD was excluded due to a failed spinal block. Thus, 47 patients (24 in Group B and 23 in Group BD) were included in the study (Fig. 1). There was no difference between the two groups in terms of their demographic data (Table 1). 


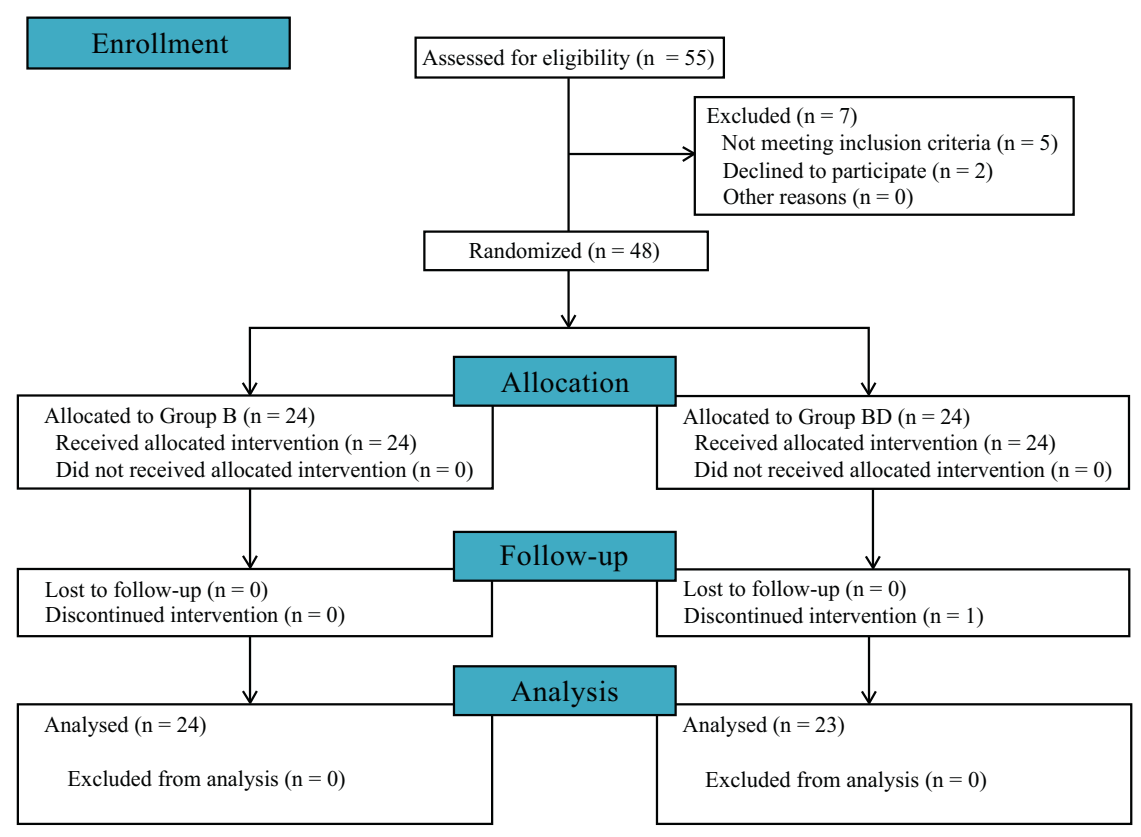

Fig. (1). CONSORT flow diagram of the study.

Table 1. Demographic data, baseline hemodynamic data, and block level.

\begin{tabular}{|c|c|c|c|}
\hline & B group $(\boldsymbol{n}=\mathbf{2 4})$ & BD group $(\boldsymbol{n}=\mathbf{2 3})$ & 0.317 \\
\hline \hline Age (yr) & $60.3 \pm 16.4$ & $55.4 \pm 16.2$ & 0.792 \\
\hline Gender (M/F) & $18 / 6$ & $62.9 \pm 11.4$ & 0.612 \\
\hline Weight (kg) & $61.2 \pm 10.5$ & $165.9 \pm 7.5$ & 0.106 \\
\hline Height (cm) & $162.2 \pm 7.8$ & $9 / 14$ & 0.454 \\
\hline ASA-PS (I/II) & $12 / 12$ & $147.0 \pm 11.9$ & 0.248 \\
\hline Baseline SBP & $142.9 \pm 12.0$ & $86.4 \pm 10.4$ & 0.090 \\
\hline Baseline DBP & $80.3 \pm 13.3$ & $101.9 \pm 9.4$ & 0.128 \\
\hline Baseline MBP & $96.6 \pm 13.5$ & $74.8 \pm 10.0$ & 0.786 \\
\hline Baseline HR & $75.8 \pm 13.8$ & $64.1 \pm 23.2$ & 0.935 \\
\hline Surgical duration (min) & $64.8 \pm 28.3$ & & 0.859 \\
\hline Surgical specialty & & 14 & \\
\hline Urology & 10 & 9 & \\
\hline Orthopedic & 14 & & \\
\hline
\end{tabular}

Data are expressed as means \pm standard deviation or number of patients.

B group: bupivacaine group; BD group: bupivacaine + dexmedetomidine group; ASA-PS: American Society of Anesthesiologists physical status; SBP: systolic blood pressure; DBP: diastolic blood pressure; MBP: mean blood pressure; HR: heart rate.

The sensory block level measured 10 min after the induction of SA was two dermatomes lower in Group BD. The motor block level as assessed using the MBS was also lower in Group BD than in Group B (Table 2). However, no patient in either group complained of intraoperative pain or required analgesics for pain control until discharge from the PACU.

The changes in SBP over time showed significant differences between the groups $(P=0.012$, Fig. 2). Assessing the differences between the groups, at time points of 5, 10, and $75 \mathrm{~min}$, the differences were statistically significant $(P=$
$0.010,<0.001$, and 0.039 , respectively). The lowest systolic, diastolic, and mean blood pressures were significantly lower in Group B than in group BD $(P=0.011,0.036$, and 0.043 , respectively; Table 2 ).

The incidence of hypotension in Group B was significantly higher than in Group BD (50.0 vs. 17.4\%, $P=0.018$; Table 2). The amount of ephedrine used to treat hypotension was significantly higher in Group B than in Group BD (median [range], 3 [0-30] vs. 0 [0-12] mg, $P=0.014$; Table 2). 
Table 2. Study data.

\begin{tabular}{|c|c|c|c|}
\hline & B group $(\boldsymbol{N}=\mathbf{2 4})$ & BD group $(\boldsymbol{N}=\mathbf{2 3})$ & 0.001 \\
\hline \hline Sensory level & $\mathrm{T} 6(\mathrm{~T} 4-\mathrm{T} 8)$ & $\mathrm{T} 8(\mathrm{~T} 6-\mathrm{T} 10)$ & 0.015 \\
\hline MBS $(0 / 1 / 2 / 3)$ & $1 / 0 / 1 / 22$ & $1 / 1 / 11 / 10$ & 0.011 \\
\hline Lowest SBP & $105.8 \pm 15.0$ & $115.7 \pm 10.1$ & 0.036 \\
\hline Lowest DBP & $58.6 \pm 11.0$ & $65.2 \pm 9.8$ & 0.043 \\
\hline Lowest MBP & $73.7 \pm 11.7$ & $79.8 \pm 8.1$ & 0.018 \\
\hline Hypotension $(N)$ & $12(50.0)$ & $4(17.4)$ & 0.014 \\
\hline Ephedrine (mg) & $3(0-30)$ & $0(0-12)$ & 0.401 \\
\hline Bradycardia $(N)$ & $9(37.5)$ & $6(26.1)$ & 0.479 \\
\hline Atropine (mg) & $0(0-0.5)$ & $700(550-1000)$ & 0.641 \\
\hline Intraoperative fluids $(\mathrm{ml})$ & $725(600-1600)$ & \\
\hline
\end{tabular}

Data are expressed as means \pm standard deviation or medians (range), except hypotension and bradycardia, which are presented as numbers of patients (\%).

B group: bupivacaine group; BD group: bupivacaine + dexmedetomidine group; MBS: modified Bromage scale $(0=$ no paralysis, $1=$ unable to raise extended leg, $2=$ unable to flex knee, 3 = unable to flex ankle); SBP = systolic blood pressure; DBP: diastolic blood pressure; MBP: mean blood pressure.

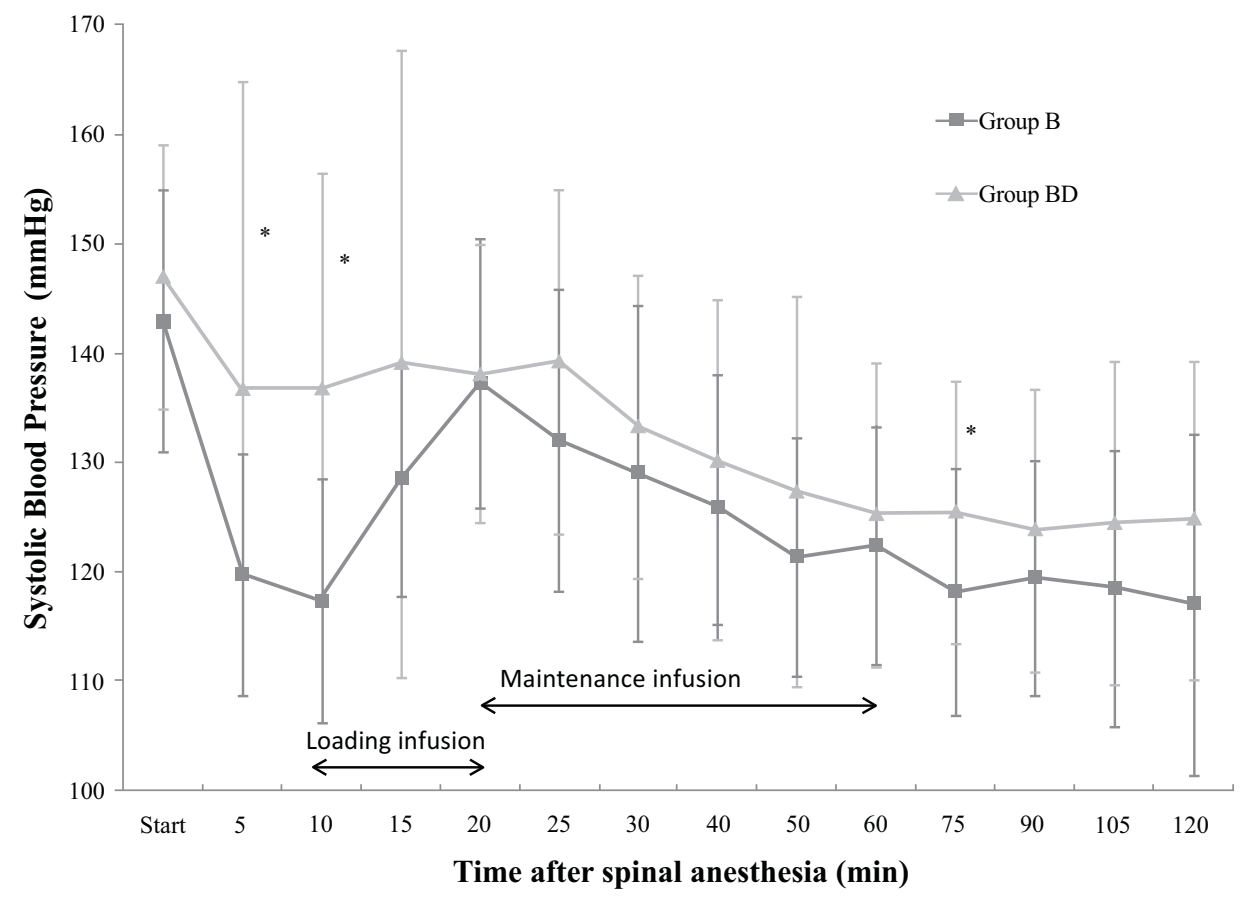

Fig. (2). Changes in systolic blood pressure for the two groups during the $120 \mathrm{~min}$ after induction of SA. Data are presented as means \pm standard deviation. ${ }^{*} P<0.05$, between groups when assessed with Student's t-test.

The baseline Ramsay sedation scales did not differ significantly between the groups (median [range], 2.5 [2-3] in Group B vs. 2 [1-3] in Group BD, $P=0.380)$. The Ramsay sedation scales increased significantly from $10 \mathrm{~min}$ after initiation of the loading dose dexmedetomidine infusion in both groups compared with baseline (Fig. 3). During the intravenous dexmedetomidine infusion, sedation was maintained adequately in both groups (median, 3 or 4 in Group B vs. 3 in Group BD) and the changes in the Ramsay sedation scale over time were not significantly different between the groups $(P=0.828$, Fig. 3).
The incidence of bradycardia and the need for atropine did not differ significantly between the groups (Table 2). Anesthesia-related adverse effects were also not significantly different between the groups (Table 3).

\section{DISCUSSION}

This study demonstrates that SA using low-dose bupivacaine $(6 \mathrm{mg})$ with dexmedetomidine $(4 \mu \mathrm{g})$ provided better hemodynamic stability, reducing the incidence of hypotension and the requirement for vasopressors, compared to SA 




Fig. (3). Changes in Ramsay sedation scales over time for the two groups. Data are presented as means. * $\mathrm{P}<0.05$, versus baseline in Group $\mathrm{B} ;{ }^{\dagger}: P<0.05$, versus baseline in Group BD.

using conventional-dose bupivacaine $(12.5 \mathrm{mg})$ at the same intravenous sedation condition. We used both ephedrine and crystalloid loading $(50-100 \mathrm{ml})$ for the correction of hypotension, but the total intraoperative volume was not different between the groups. It appears that a relatively small loading volume did not produce a statistically significant difference between the groups.

Table 3. Adverse effects.

\begin{tabular}{|c|c|c|}
\hline & B group $(\boldsymbol{N}=\mathbf{2 4})$ & BD group $(\boldsymbol{N = 2 3 )}$ \\
\hline \hline Dry mouth & $8(33.3)$ & $6(26.1)$ \\
\hline Nausea & $3(12.5)$ & $2(8.7)$ \\
\hline Vomiting & $1(4.2)$ & 0 \\
\hline Pruritus & $1(4.2)$ & 0 \\
\hline Headache & $1(4.2)$ & 0 \\
\hline Dyspnea & $1(4.2)$ & 0 \\
\hline Respiratory depression & $1(4.2)$ & 0 \\
\hline
\end{tabular}

Data are expressed as numbers of patients (\%). Respiratory depression was defined as respiratory rate $\mathrm{min}^{-1}<8$. All adverse effects did not showed significant difference between the groups

As mentioned earlier, several studies have evaluated the incidence of hypotension after SA using low-dose spinal bupivacaine with opioids, but few studies have compared low-dose intrathecal bupivacaine plus dexmedetomidine with a conventional dose of bupivacaine in terms of the hemodynamic effects. Unlike intrathecal opioids, intrathecal dexmedetomidine itself can produce hypotension, and the optimal intrathecal dexmedetomidine dose has not been established [10]. In our study, the intrathecal doses of bupivacaine $(6 \mathrm{mg})$ and dexmedetomidine $(4 \mu \mathrm{g})$ in Group BD were set according to those in previous studies; Kim et al. [11] showed that $6 \mathrm{mg}$ of bupivacaine resulted in a median sensory block level of $\mathrm{T} 10$ at a mean of 10 min after spinal injection. The addition of 3-5 $\mu \mathrm{g}$ of dexmedetomidine to the intrathecal local anesthetics effectively extended the duration of the motor and sensory block $[7,11,12]$.

In the present study, we chose the use of intravenous dexmedetomidine, not midazolam or propofol, for sedation during SA because dexmedetomidine can be effectively used for sedation during regional anesthesia; moreover, unlike other sedative agents, dexmedetomidine also has an analgesic effect when used intrathecally. Therefore, dexmedetomidine can replace other intravenous sedative agents or intrathecal analgesics.

The optimal loading and maintenance doses of dexmedetomidine for sedation have been somewhat controversial [13]. Kaya et al. [14] reported that a loading dose of 0.5 $\mu \mathrm{g} / \mathrm{kg}$ over $10 \mathrm{~min}$ provided adequate sedation during bupivacaine SA. Ickeringill et al. [15] reported that omitting the loading dose and using a maintenance dose of $0.2-0.4$ $\mu \mathrm{g} / \mathrm{kg} / \mathrm{h}$ avoided undesirable hemodynamic effects and provided satisfactory sedation. Given this background, a loading dose of $0.5 \mu \mathrm{g} / \mathrm{kg}$ for $10 \mathrm{~min}$, and a maintenance dose of 0.2 $\mu \mathrm{g} / \mathrm{kg} / \mathrm{h}$ for $40 \mathrm{~min}$, were administered in this study. Although we infused the same dose of intravenous dexmedetomidine in both groups, the level of sedation may be different according to the level of sensory block induced by spinal anesthesia $[16,17]$. Thus, we assessed the level of sedation using the Ramsay sedation scale. These loading and maintenance doses resulted in adequate sedation in both groups. 
Although hypotension is a common side effect of intravenous dexmedetomidine infusion, transient hypertension due to $\alpha-2 \mathrm{~B}$ receptor stimulation, which causes vasoconstriction, may also occur, especially during a loading infusion that causes rapid and high blood concentrations of dexmedetomidine $[18,19]$. In the present study, at $10 \mathrm{~min}$ after the loading infusion, the mean SBP in Group B increased by $\sim 20$ mmHg (Fig. 2). However, we do not believe that this transient hypertension was related to the rapid and large loading dose; rather, it can be explained as follows. In Group B, after the induction of SA, the SBP in most patients dropped significantly; thus, they received an ephedrine bolus and fluids according to our hypotension management protocol. Also, no such transient hypertension was observed in Group BD, which received intravenous dexmedetomidine under the same infusion rates as in Group B.

Unlike the use of intravenous dexmedetomidine, the use of intrathecal dexmedetomidine is relatively rare and few studies have been performed in human subjects $[7,11,12$, 20]. The mechanisms underlying the analgesic action of intrathecal dexmedetomidine have not been established. There are $\alpha_{2}$-adrenoreceptors located on the primary afferent terminals of neurons in the spinal cord, brainstem, and peripheral tissues [21]. Thus, $\alpha_{2}$-agonists may trigger analgesic effects by acting at these sites. The intrathecal administration of $\alpha_{2}$ agonists provides more potent analgesia than systemic administration [22]. Also, they have synergistic effects with intrathecal local anesthetics and an analgesic-sparing effect in postoperative pain control [23-25]. In terms of sedative effects, intrathecal clonidine, another $\alpha_{2}$-agonist, showed dose-dependent sedative effects when administered intrathecally [26]. However, intrathecal dexmedetomidine did not show significant sedative effects (Ramsay sedation scale $>3$ ) at the doses used clinically $(3-10 \mu \mathrm{g})[7,24,25]$.

Although the reported incidence of hypotension after SA varies considerably (from 15.3 to $33 \%$ ) due to the use of different definitions in various reports, it is clear that hypotension is one of the most common and clinically meaningful complications of SA [1, 27]. In the present study, the incidence of hypotension was $17.4 \%$ in Group $\mathrm{BD}$, despite a stricter hypotension definition than those used in other studies $[1,7,25]$ and intravenous sedation.

The incidence of bradycardia in both groups in the present study was higher than predicted. The incidence of bradycardia following SA and the administration of intravenous dexmedetomidine has been reported to be $10-15 \%$ [27]. We suggest that the high incidence of bradycardia in our study may have been affected by the reduced plasma catecholamine levels due to the infusion of dexmedetomidine in addition to enhanced vagal activity caused by SA [5].

The present study has an important limitation. We did not measure the time of regression from the sensory and motor block. Thus, we do not know the maximum duration for which adequate analgesia and motor block for surgery can be provided. In our study, two patients in Group BD had operation times of $\sim 120 \mathrm{~min}$, but neither complained of surgical pain or discomfort. However, additional studies should be performed with larger numbers of patients to guarantee adequate analgesic effects.

In conclusion, low-dose bupivacaine $(6 \mathrm{mg})$ with dexmedetomidine $(4 \mu \mathrm{g})$ SA caused less hypotension and reduced the need for vasopressors in comparison to a conventional dose of bupivacaine $(12 \mathrm{mg})$ alone for SA.

\section{CONFLICT OF INTEREST}

The authors confirm that this article content has no conflict of interest.

\section{ACKNOWLEDGEMENTS}

Declared none.

\section{REFERENCES}

[1] Tarkkila P, Isola J. A regression model for identifying patients at high risk of hypotension, bradycardia and nausea during spinal anesthesia. Acta Anaesthesiol Scand 1992; 36: 554-8.

[2] Ben-David B, Frankel R, Arzumonov T, Marchevsky Y, Volpin G. Minidose bupivacaine-fentanyl spinal anesthesia for surgical repair of hip fracture in the aged. Anesthesiology 2000; 92: 6-10.

[3] Olofsson C, Nygards EB, Bjersten AB, Hessling A. Low-dose bupivacaine with sufentanil prevents hypotension after spinal anesthesia for hip repair in elderly patients. Acta Anaesthesiol Scand 2004; 48: 1240-4.

[4] Chaney MA. Side effects of intrathecal and epidural opioids. Can J Anaesth 1995; 42: 891-903.

[5] Bhana N, Goa KL, McClellan K. Dexmedetomidine. Drugs 2000; 59: 263-8.

[6] Elcicek K, Tekin M, Kati I. The effects of intravenous dexmedetomidine on spinal hyperbaric ropivacaine anesthesia. J Anesth 2010; 24: 544-8.

[7] Kanazi GE, Aouad MT, Jabbour-Khoury SI, et al. Effect of lowdose dexmedetomidine or clonidine on the characteristics of bupivacaine spinal block. Acta Anaesthesiol Scand 2006; 50: 2227.

[8] Bromage PR, Burfoot MF, Cromwell DE, Pettigrew RT. Quality of epidural blockade. I: Influence of physical factors. Br J Anaesth 1964; 36: 342-52.

[9] Ramsay MAE, Savege TM, Simpson BRJ, Goodwin R. Controlled sedation with alphaxalone-alphadolone. BMJ 1974; 2: 656-9.

[10] Eisenach JC, Tong CY. Site of hemodynamic effects of intrathecal alpha 2-adrenergic agonists. Anesthesiology 1991; 74: 766-71.

[11] Kim JE, Kim NY, Lee HS, Kil HK. Effects of intrathecal dexmedetomidine on low-dose bupivacaine spinal anesthesia in elderly patients undergoing transurethral prostatectomy. Biol Pharm Bull 2013; 36: 959-65.

[12] Gupta R, Verma R, Bogra J, Kohli M, Raman R, Kushwaha JK. A comparative study of intrathecal dexmedetomidine and fentanyl as adjuvants to bupivacaine. J Anaesth Clin Pharmacol 2011; 27: 33943.

[13] Gerlach AT, Dasta JF. Dexmedetomidine: an updated review. Ann Pharmacother 2007; 41: 245-52.

[14] Kaya FN, Yavascaoglu B, Turker G, et al. Intravenous dexmedetomidine, but not midazolam, prolongs bupivacaine spinal anesthesia. Can J Anaesth 2010; 57: 39-45.

[15] Ickeringill M, Shehabi Y, Adamson H, Ruettimann U. Dexmedetomidine infusion without loading dose in surgical patients requiring mechanical ventilation; haemodynamic effects and efficacy. Anaesth Intensive Care 2004; 32: 741-5.

[16] Gentili M, Huu PC, Enel D, Hollande J. Bonnet F. Sedation depends on the level of sensory block induced by spinal anaesthesia. Br J Anaesth 1998; 81: 970-1.

[17] Iida R, Iwasaki K, Kato J, Ogawa S. Bispectral index is related to the spread of spinal sensory block in patients with combined spinal and general anaesthesia. Br J Anaesth 2011; 106(2): 202-7.

[18] Maze M, Scarfini C, Cavaliere F. New agents for sedation in the intensive care unit. Crit Care Clin 2001; 17: 881-97. 
[19] Bloor BC, Ward DS, Belleville JP, Maze M. Effects of intravenous dexmedetomidine in humans. II. Hemodynamic changes. Anesthesiology 1992; 77: 1134-42.

[20] Al-Ghanem SM, Massad IM, Al-Mustafa MM, et al. Effect of adding dexmedetomidine versus fentanyl to intrathecal bupivacaine on spinal block characteristics in gynecological procedures: A double blind controlled study. Am J Appl Sci 2009; 6: 882-7.

[21] Unnerstall JR, Kopajtic TA, Kuhar MJ. Distribution of alpha 2 agonist binding sites in the rat and human central nervous system: analysis of some functional, anatomic correlates of the pharmacologic effects of clonidine and related adrenergic agents. Brain Res 1984; 319: 69-101.

[22] Eisanach JC, De Kock M, Klimscha W. alpha(2)-adrenergic agonists for regional anesthesia. A clinical review of clonidine (19841995). Anesthesiology 1996; 85: 655-74.

[23] Strebel S, Gurzeler J, Schneider M, Aeschbach A, Kindler C. Small-dose intrathecal clonidine and isobaric bupivacaine for or- thopedic surgery: a dose-response study. Anesth Analg 2004; 99: 1231-8.

[24] Mohamed AA, Fares KM, Mohamed SA. Efficacy of intrathecally administered dexmedetomidine versus dexmedetomidine with fentanyl in patients undergoing major abdominal cancer surgery. Pain Physician 2012; 15: 339-48.

[25] Al-Mustafa MM, Abu-Halaweh SA, Aloweidi AS, et al. Effect of dexmedetomidine added to spinal bupivacaine for urological procedure. Saudi Med J 2009; 30: 360-70.

[26] Filos KS, Goudas LC, Patroni O, Polysou V. Hemodynamic and analgesic profile after intrathecal clonidine in humans: A doseresponse study. Anesthesiology 1994; 81: 591-601.

[27] Carpenter RL, Caplan RA, Brown DL, et al. Incidence and risk factors for side effects of spinal anesthesia. Anesthesiology 1992; 76: $906-16$.

Received: August 16, 2015

Revised: November 11, 2015

Accepted: November 17, 2015

(C) Chang et al.; Licensee Bentham Open.

Articles are licensed under the terms of the Creative Commons Attribution-NonCommercial 4.0 International Public License (CC BY-NC 4.0) (https://creativecommons.org/licenses/by-nc/4.0/legalcode), which permits unrestricted, non-commercial use, distribution and reproduction in any medium, provided that the work is properly cited. 